\title{
Procesos logísticos de un centro nacional de repuestos, mediante técnicas de simulación y almacenamiento LEAN
}

\author{
Logistic processes of a national spare parts center, using LEAN storage \\ and simulation techniques
}

\author{
Carlos Casadiego A. \\ carloscasadiego14@gmail.com \\ María L. Mapelli S. \\ Universidad de Carabobo, Venezuela
}

Artículo recibido septiembre 2018 | Arbitrado en octubre 2018 | Publicado en enero 2019

\section{RESUMEN}

El presente artículo tiene como mostrar una propuesta soluciones de mejoras en los procesos logísticos de un almacén de autopartes. La metodología empleada fue a través de un modelo de simulación construido en el programa ARENA $®$, para así evaluar y determinar las actividades que podrían ser mejoradas y estimar el impacto de las distintas propuestas en el entendimiento del sistema en estudio. Posteriormente, se selecciona la configuración más conveniente, complementando con la filosofía Lean. Se llega a la conclusión de que la mejor configuración para aumentar el desempeño del almacén es anexar un elevador entre el almacén B y C, adquisición de dos (2) montacargas, surtido semanal y mensual de los almacenes B y C, respectivamente. Finalmente, se realiza una redistribución de operadores para balancear la carga de trabajo entre ellos, siendo los resultados esperados del almacén, que el tiempo promedio que tarda un pedido desde que llega hasta que se despacha sea de 5,966 días, un $5,43 \%$ de los pedidos se despachen incompletos y el $14,693 \%$ de los pedidos se despachen después de 15 días.

Palabras clave: Filosofía Lean; técnicas de simulación; proceso logístico, almacén de autopartes

\section{ABSTRACT}

The present article has as a proposal for solutions for improvements in the logistics processes of an auto parts warehouse. The methodology used was through a simulation model built in the ARENA® program, in order to evaluate and determine the activities that could be improved and estimate the impact of the different proposals on the understanding of the system under study. Subsequently, the most convenient configuration is selected, complementing with the Lean philosophy. It is concluded that the best configuration to increase the performance of the warehouse is to attach an elevator between warehouse $\mathrm{B}$ and $\mathrm{C}$, acquisition of two (2) forklifts, weekly and monthly assortment of warehouses B and C, respectively. Finally, a redistribution of operators is carried out to balance the workload between them, with the expected results of the warehouse being that the average time it takes for an order from the time it arrives until it is dispatched is 5,966 days, $5.43 \%$ of orders are dispatched incomplete and $14.693 \%$ of orders are dispatched after 15 days.

Key words: Lean philosophy; simulation techniques; logistics process, auto parts warehouse 


\section{INTRODUCCIÓN}

Actualmente, es vital proveer a los clientes un buen servicio post-venta del producto, ya que es valorado por el consumidor a la hora de seleccionar un vehículo (Medina y José, 2012). Es importante ofrecerle al cliente el producto requerido, en el momento y con las cantidades y especificaciones solicitadas, de lo contrario buscaran otras opciones. La Corporación Automotriz ZGT, tiene como meta alcanzar el liderazgo en el sector automotriz de Venezuela, para ello es importante mantener la búsqueda constante de oportunidades de mejora. En este sentido, son cada vez más conscientes de la importancia de la gestión de almacenes (Espinal, Montoya, y Arenas, 2010) y la gestión logística en general (Olivos, Carrasco, Flores, Moreno, y Nava, 2015) como parte esencial para determinar el éxito de sus productos o servicios

La gestión de almacenes es un factor importante dentro de la red logística de las organizaciones, ya que constituyen decisiones claves que definen en gran medida la estructura de costos y servicios de una empresa. Mediante un apropiado almacenamiento se logra obtener resultados en el aumento de beneficios, ya que permite una mejor distribución de las mercancías, mejora en los procesos y actividades del almacén. De esta manera asegurar disponer de las cantidades requeridas y en el momento oportuno, procurando amortiguar el desequilibrio existente entre oferta y demanda, reducir costos, aprovechar al máximo el espacio y acceder a los ítems almacenados en el mercado. Ésta constituye decisiones claves que definen en gran medida la estructura de costos y servicios de una empresa. Mediante un apropiado almacenamiento se logra obtener resultados en el aumento de beneficios, ya que permite una mejor distribución de las mercancías, mejora en los procesos y actividades del almacén, de esta manera asegurar disponer de las cantidades requeridas $y$ en el momento oportuno, procurando amortiguar el desequilibrio existente entre oferta $y$ demanda, reducir costos, aprovechar al máximo el espacio y acceder a los ítems almacenados.

En la investigación se estudia un centro nacional de repuestos y se elaboraron propuestas para mejorar su tiempo de respuesta, mejora en el porcentaje de pedidos despachados con todos los ítems solicitados por el cliente y el empleo de sus diferentes recursos. En el análisis se empleó la filosofía Lean (Díaz, Rivera, y Guerra, 2014) (López, Grajales, Corrales, 2017) (Araújo, 2011) para la identificación de todos los desperdicios presentes en el sistema y se construyó un modelo de simulación para comprender su funcionamiento, identificar las actividades susceptibles a mejoras y estimar el impacto que tienen las diferentes propuestas de mejora en el rendimiento del almacén, seleccionando así la configuración del sistema más conveniente.

\section{METODOLOGÍA}

Para efectos de esta investigación el estudio se realiza en las instalaciones de la Corporación Automotriz Z.G.T. Ésta dispone de tres (3) almacenes que conforman el Centro Nacional de Repuestos de la marca Chery Automovile y Grand Tiger.

Como fuentes y técnicas de recolección de información los analistas utilizan una variedad de métodos a fin de recopilar datos sobre la situación existente, cada uno tiene sus ventajas, entre ellos se encuentran cuestionario, inspección de 
registro, observación y entrevista. Entre las técnicas que se utilizaron para el desarrollo de la investigación en la obtención de los datos presentes son:

a) Observación Directa: Para este estudio en particular a través de la observación directa, se le hizo seguimiento a todas las operaciones realizadas por el personal dentro del almacén incluyendo las operaciones administrativas, de esta manera detectar las actividades que requieren de mejoras. Este comprende cada área del almacén para analizar la manera en cómo se encuentra el inventario y obtener la situación actual del sistema de manejo de materiales (Crotte, 2011) .

b) Entrevista no estructurada: Las entrevistas se utilizaron para recabar información en forma verbal, a través de preguntas que propone el analista. Quienes responden pueden ser gerentes o empleados, los cuales son usuarios actuales del sistema existente o aquellos que proporcionan datos o serán afectados por la aplicación de la propuesta. Estas entrevistas no estructuradas se realizaron con las personas involucradas directamente en los almacenes, a fin de recolectar información necesaria para el desarrollo del presente trabajo de investigación (Díaz-Bravo, et al., 2013).

c) Investigación de los registros históricos de la empresa: Conocer la cantidad de material que se almacena, el proceso de la empresa, su número de partes, el flujo de los materiales, disponibilidad de equipos, entre otros (Fernández, 2002).

Las técnicas de procesamiento y análisis de los datos para el sistema en estudio, se desarrolla un modelo de simulación en el programa ARENA (Ré, Arena, y Giubergia, 2012) (Casadiego y Mapelli, 2017). Este programa se presenta como una Herramienta "Orientada al Proceso", por cuanto permite la descripción completa de la experiencia que una entidad desarrolla al interior del sistema conforme fluye. En efecto durante el modelamiento se pueda observar la definición de eventos, cálculos de variables, actualización de lista de eventos, avance del reloj de simulación, entre otro, están presentes "tras bambalinas" siendo todas ellas desarrolladas internamente por el software. Posteriormente, para el estudio de las variables de entrada del modelo de simulación se utiliza el programa STATGRAPHICS (Trujillo, Llinás, Obeso, y Rojas, 2005), donde se realiza el histograma de frecuencia para obtener una idea de cuál tipo de distribución de probabilidades se puede ajustar, corroborando la hipótesis con la prueba de bondad de ajuste Kolmogorov-Smirnov o $\mathrm{X}^{2}$ (Pedrosa, 2015).

\section{Fase I: Diagnóstico de la situación actual}

Esta fase inicia observando las diferentes actividades que se llevan a cabo en el Centro Nacional de Repuestos, además se verifico los diferentes repuestos que se manejan, la distribución de la organización y los equipos móviles que dispone. También se verificaron los diversos diagramas de flujos de todas las actividades que se desarrollan en los almacenes, sumado a eso se recolectará información referente a la demanda, los clientes que maneja la organización y la forma en que almacena sus productos. 


\section{Fase II: Análisis de la situación actual}

Ya entendido el funcionamiento del Centro Nacional de Repuestos, se procedió a realizar estudios de tiempo para cuantificar el tiempo que tardan los operadores en desarrollar las actividades (armado de pedidos, auditorias, despachos y recepción), realizando flujogramas para el análisis de todos los procesos del almacén. Mediante el análisis de la data histórica se logró analizar variables como la demanda, de esta manera se pudo determinar y cuantificar los desperdicios presentes.

\section{Fase III: Construcción del modelo de simulación}

En esta fase se pasaron de los datos físicos obtenidos en la recolección de datos a la implementación de un modelo de simulación en el programa ARENA, para obtener un modelo que se ajuste a los datos recolectados. Se procedió a generar todos los procesos que se encuentran en el centro nacional de repuestos, desde la alimentación de los diversos almacenes, llegadas de pedidos hasta la salida de los productos a los diferentes concesionarios. Para las variables elegidas, se realizó la prueba de bondad de ajuste en el programa STATGRAPHICS, para determinar la distribución de probabilidades que se ajuste a cada una de ellas y obtener los datos de entrada. Posteriormente se validó y verifico el modelo, para asegurarse de que sea la representación correcta de las actividades realizadas en el almacén, para así detectar las actividades que acarreen posibles mejoras.

\section{Fase IV: Establecer acciones orientadas a la filosofía lean}

En esta fase se realizó un estudio en los distintos procesos ejecutados en el
Centro Nacional de Repuestos con la finalidad de detectar aquellas actividades que no agreguen valor a la organización e implementar acciones orientadas a su eliminación mediante el uso de la filosofía lean.

\section{Fase V: Diseño de las propuestas de mejoras}

Una vez diagnosticados $\mathrm{y}$ jerarquizados todos los problemas que se encuentran dentro del Centro Nacional de Repuestos, se obtuvo un análisis con diferentes alternativas, con la finalidad de mejorar los procesos logísticos como resultado de aplicar la filosofía "lean" en las diferentes áreas del centro nacional de repuestos.

\section{RESULTADOS}

El Centro Nacional de Repuestos se encuentra ubicado en el estado Carabobo, municipio San Diego. Posee un terreno con $20.571 \mathrm{~m}^{2}$, en el cual alberga tres (3) almacenes, un (1) edificio administrativo, un (1) comedor, un (1) estacionamiento corporativo y el personal y clientes, un (1) área de reuniones, el área de servicio técnico, el área de lockers y anexo comparte un espacio con la Corporación Trasuca

El almacén A cuenta con un área de $2.164 \mathrm{~m}^{2}$, dispone de 39 zonas para el almacenamiento de mercancía, distribuidas en nueve pasillos, cuenta con el almacén no productivo, la oficina de control de inventario, sanitarios, la zona de hierros y la zona de motores. Este almacén cuenta con 189 Racks donde 88 son de cuatro niveles, 80 de tres niveles y 21 de dos (2) niveles, tiene un portón de 3,6 $\mathrm{m}$ de largo y 2,5 $\mathrm{m}$ de alto, por donde pasa la mercancía para alimentación de los almacenes B y C, así 
como para la recepción de las cajas provenientes de China, las cuales son recibidas en el Almacén B, donde se realiza el proceso de auditoría correspondiente.

El almacén B cuenta con un área de $2388 \mathrm{~m}^{2}$, se constituye básicamente de cuatro (4) zonas, área de preparación de pedidos, mezzanina, jaula de valores y oficina de logística. El área de material de hierros cuenta con $101.1 \mathrm{~m}^{2}$ destinado al resguardo de las piezas metálicas de gran volumen y peso, dispone de 16 racks, donde dos (2) son de tres (3) niveles y 12 son de dos (2) niveles, arreglados en tres (3) pasillos de 1,1; 2 y 1,22 m, respectivamente.

El almacén $C$ cuenta con un área de $1.167 \mathrm{~m}^{2}$, dispone de 11 zonas para el resguardo de mercancía, distribuidas en siete pasillos. Este almacén se compone generalmente en tres áreas, destinadas a la custodia de las piezas de carrocería, vidrio y parachoques. Este almacén cuenta con 144 Racks, tiene un portón de 3,2m de largo y $2,5 \mathrm{~m}$ de alto, por donde pasa la mercancía para alimentación del almacén $\mathrm{B}$, así como para la recepción de las piezas enviadas por el almacén A para la reposición del almacén.

Se cuenta con un personal operativo de 15 operadores de materiales que son los responsables del armado de pedidos, surtido, orden y limpieza del almacén, dos montacarguistas para el manejo de cargas, un jefe de logística, cuatro supervisores de planta, una jefa de control de inventarios, tres analistas de inventario y dos pasantes INCES, que son los responsables de proteger los inventarios de repuestos, que posteriormente serán comercializados $\mathrm{y}$ distribuidos a los diferentes concesionarios, distribuidores y talleres autorizados, y velar que en todo momento el almacén debe operar apegándose a principios establecidos por la empresa, con el fin de evitar retrasos en entregas, extravíos, hurtos o deterioro de los materiales. Generalmente se distribuyen dos operadores de materiales para el proceso de auditoría, tres para realizar actividades de surtido, además realizar actividades de orden y limpieza, cuatro para el armado de pedido, tres que soporten las actividades del almacén $\mathrm{A}$, otros tres para soportar las actividades del almacén C. Un montacarguista para el almacén A y otro para el almacén $B$, este último también deberá realizar las actividades de manejo de cargas del almacén $C$, los supervisores de área son dos para el almacén $\mathrm{B}$, uno para el $\mathrm{A}$ y el restante se encarga del almacén $C$.

De los resultados obtenidos por el modelo de simulación (Casadiego y Mapelli, 2017) se obtiene que el centro nacional de repuestos despacha en promedio 16.514 pedidos al día, y el tiempo que tarda un pedido desde que se recibe hasta que se despacha es 13.619 días; destacando que en promedio $34.485 \%$ de los pedidos se despachan después de 15 días y 9.313\% pedidos tuvieron que ser modificados ya que no se disponía del número de parte solicitado.

Mediante los resultados se puede evidenciar la carga de trabajado en los operadores, la cual ratifica el desbalance existente en la misma. Se observa que los auditores se mantienen ocupados el 79.62\% del tiempo, mientras que los operadores responsables del almacén $\mathrm{C}$ se mantienen ocupados solo el $25.38 \%$ del tiempo. Esta situación queda en evidencia mediante los cuellos de botellas que se visualizan a través de los resultados se confirma que las actividades están mal distribuidas.

La utilización de los dos montacargas que realizan actividades en el almacén $A$ es de $80.08 \%$, de los dos montacargas que realizan las asignaciones del almacén $B$, es 
de 68.726\% del tiempo; para el almacén C, el montacargas tuvo una utilización 43.64\%. Este porcentaje de utilización detallado se considera alto y se ve reflejado en demoras, ya que diversas actividades dependen del uso de este equipo como, recepción y ubicación de contenedores, despacho de mercancía entre almacenes, en actividades de despacho y auditoria, en caso del equipo estar ocupado y ser requerido en otra actividad; se debe esperar a que se desocupe.

\section{Propuestas de mejoras}

El almacén $\mathrm{C}$ es el encargado de alimentar al almacén B en piezas de carrocería, vidrios y parachoques, debido al volumen de las piezas el surtido entre dichos almacenes se realiza a contra pedido y diariamente. Dicho almacén, se encuentra anexo al almacén B a una altura de 3.6 metros por encima del nivel del piso del almacén B.

Actualmente el centro de nacional de repuesto incurre en incumplimiento de pedidos entregados en un lapso menor de 15 días, el $31.53 \%$ se entregan después de dicho lapso de tiempo. Al despachar la mercancía desde almacén $\mathrm{C}$ hacia el $\mathrm{B}$, el montacargas debe recorre aproximadamente 410 metros y demora 11 minutos realizando este proceso, afectando esto el tiempo que se tarda en preparar un pedido un pedido y por ende afectando en el tiempo de repuesta de la organización; cabe destacar que los montacargas se mantienen ocupados $64.148 \%$ del tiempo (según los resultados de simular la situación actual), perjudicando el desempeño oportuno de las actividades del centro nacional de repuestos.

Por lo tanto, para disminuir el desperdicio de transporte que está presente, se propone colocar un elevador que conecte los dos almacenes para de esta manera disminuir el uso del montacargas asignado al almacén C; y así se espera reducir los tiempos y distancias asociadas al traslado de mercancía.

Se usó el modelo de simulación para estimar el impacto que tendría en el desempeño del almacén la propuesta y los resultados se muestran en (Casadiego y Mapelli, 2017). Se puede apreciar que la propuesta tiene un impacto significativo en el porcentaje de utilización del montacargas del almacén $\mathrm{C}$ con una disminución del 14.09\%; el porcentaje de utilización de los operadores del almacén $\mathrm{C}$ disminuye en un 9.45\%; el porcentaje de pedidos con un tiempo de respuesta mayor a 15 días disminuye en un $9.015 \%$ y tiempo que tarda en ser atendida una solicitud desde que llega al almacén C hasta que es respondida (mejora en un 0.806 días); en el resto de las variables de interés la diferencia no es significativa. Este elevador estará ubicado diagonal a la zona de motor y transmisores, al lado del pasillo 19.

Este elevador tiene conexiones eléctricas resistentes a la intemperie, sellan el agua y la suciedad; la unidad de potencia proporciona un funcionamiento hidráulico suave y silencioso, fue seleccionado por simplicidad, bajo costo, y cumple con los requisitos mínimos para realizar el transporte de los distintos repuestos y cajas que se manejan en los almacén, en la figura 32 se muestra el elevador propuesto y en el anexo 8 los planos del mismo. Para finalizar, esta propuesta tendrá un impacto positivo en el porcentaje de pedidos con un tiempo de repuesta mayor a 15 días, tiempo que tarda en ser atendida una solicitud desde que llega al almacén $\mathrm{C}$ hasta que es respondida, y eliminando el desperdicio de transporte, ya que si no se considera dicha propuesta, el montacargas debe recorrer aproximadamente 410 metros y demora 11 minutos realizando este proceso. 
El montacargas es uno de los recursos más importantes en el centro nacional de repuestos debido al gran volumen de piezas y cajas que se manejan, siendo esto la consecuencia de que el porcentaje de utilización de este recurso sea alto. El almacén A, presenta un porcentaje de utilización de montacargas del 80\%, mientras que los montacargas del almacén B tiene un $70 \%$ de utilización, y el porcentaje de utilización del montacargas del almacén C es del 43\%. Actualmente el centro nacional de repuestos existen dos montacargas en el almacén $A$, dos montacargas en el almacén $B$ y uno en el almacén C.

Este porcentaje de utilización de $80 \%$ se considera alto en el almacén $\mathrm{A}$, y se debe a que estos montacargas realizan la búsqueda de piezas para el surtido del almacén $\mathrm{C}$ y $\mathrm{B}$, para cuando llega un contenedor, y diariamente se utilizan los montacargas para la búsqueda de piezas en dicho almacén para completar pedidos, teniendo esta última actividad repercusión importante con el tiempo de respuesta de los pedidos. Y las actividades que se realizan con los montacargas del almacén B son preparación de pedido, surtido del almacén B, recepción de contenedores, auditoria y despacho, por ende la mayoría parte del tiempo de la jornada laboral se mantienen ocupados. Debido al uso frecuente de los montacargas para desempeñar las diferentes actividades de los almacenes, se registran desperdicios de tiempo de 25 minutos, en el área de armado de pedidos, donde los operadores se ven obligados a retrasar sus operaciones ya que requieren el uso del equipo móvil para el manejo de paletas o cargas pesadas. El modelo de simulación, se utilizó para evaluar el impacto que tiene en el desempeño de los almacenes al adquirir montacargas, asignarlos al almacén A y B respectivamente.

Otro aspecto donde se detectó mejora fue en el tiempo que tardan buscando y enviando un pedido a auditoria, así como en el tiempo que tardo un pedido en despacho; evaluando estas variables, nuevamente el escenario dos brindo resultados favorables; obteniendo reducción de 0.208 y 3.304 días respectivamente en dichas variables; de igual forma el escenario tres ofrece un panorama positivo, reduciendo el tiempo que tarda un pedido en despacho en 2.298 días, sin embargo al comparar con el escenario dos, este escenario incurrió en un aumento en el tiempo que tarda un pedido en ser buscado y enviado a auditoria 0.224 .

El porcentaje de pedidos con tiempo de repuesta mayor a 15 días, disminuyo en $15.73 \%$ en el escenario dos, en los otros escenarios dicha variable obtuvo una disminución hasta de 6\%. La utilización de los montacargas actualmente es de $64 \%$, a través de los escenarios simulados se detectó una reducción en los porcentajes de utilización de los montacargas, lo que permitió agilizar procesos y reducir cuellos de botella; el escenario con mejor resultados fue el dos con una reducción de $20.73 \%$.

\section{CONCLUSIONES}

Las problemáticas del centro nacional de repuestos se basa principalmente en la información de surtido continuo de piezas en el almacén $B$ que no se encuentra definida de manera adecuada, por lo tanto el armado de pedidos se ve afectado, siendo esto causa de que el $31.53 \%$ de las veces se entregue un pedido después de 15 días, sumado a esto existen operadores encargados del armado de pedidos que trabajan el 35\% del tiempo, mientras que otros se mantienen ocupados el $80 \%$ de la 
jornada laboral, además, los equipos móviles en múltiples ocasiones retrasan las actividades, ya que los equipos se encuentran ocupados realizando diferentes labores, esta situación trae como consecuencia retrasos de hasta dos días entre despachos de mercancía entre almacenes. Estas actividades mencionadas anteriormente, ocasionan desperdicios como inventario, transporte, conductual, demora y movimiento, logrando esto que el tiempo de repuesta del centro nacional de repuesto Chery sea aún mayor.

La simulación de sistemas, se utilizó como herramienta para el análisis de la situación actual de los almacenes, para detectar aquellas actividades susceptibles a mejoras, así como poder evaluar el impacto de las propuestas de mejoras planteadas, en lo que respecta a las actividades de recepción, almacenamiento y despacho, obteniendo la configuración adecuada para prestar el servicio de una forma apropiada.

Cada una de las propuestas descritas, tuvo impacto en variables específicas de salidas. La mejor configuración que se logró obtener del modelo de simulación fue el escenario que plantea las propuestas del elevador y la adquisición de dos montacargas, uno para que brinde soporte en las operaciones del almacén $\mathrm{A}$ y el otro será asignado en las operaciones del B. En dicha configuración se considera el surtido semanal del almacén B y el surtido mensual del C, otro cambio asociado en esta configuración respecto a la situación actual está referido a los operadores encargados del C quienes brindaran soporte a los del A y el $\mathrm{B}$ en las actividades involucradas de surtido y finalmente se realizó una redistribución de operadores para balancear la carga de trabajo.

Con la configuración descrita anteriormente, el tiempo que tarda un pedido desde que llega hasta que se despacha es de 5.966 día, al menos el $14,693 \%$ de estos se entregaran después de 15 días; el número de pedidos que se despachan al día es de 20,942 y se requiere de una fuerza laboral de 14 operadores que se redistribuirán de la siguiente manera: tres operadores trabajaran en el almacén $A$, uno en el almacén $\mathrm{C}$, uno en surtido, cinco en búsqueda $\mathrm{y}$ armado de pedidos y 4 operadores en auditoria y despacho.

Para evaluar el rendimiento del almacén, las variables de salidas más importantes del modelo son el tiempo que tarda un pedido desde que llega hasta que se despacha, el porcentaje de pedidos que se despachan en más de 15 días, el porcentaje de pedidos despachados incompletos, porcentaje de utilización de los montacargas y el porcentaje de tiempo de ocupación de cada uno de los operadores de los tres almacenes.

\section{REFERENCIAS}

Araújo, P. (2011). "Universidades Lean": construcción para la reflexión. Revista de la educación superior, 40(160), 135155

Casadiego, C., y Mapelli, M. (2017). Propuesta de mejora en los procesos logísticos de un centro nacional de repuestos, mediante el uso de técnicas de simulación y alamcenamiento Lean. Valencia: Universidad de Carabobo

Crotte, I. (2011). Elementos para el diseño de técnicas de investigación: una propuesta de definiciones y procedimientos en la investigación científica. Tiempo de educar, 12(24), 277-297

Díaz, P., Rivera, O., y Guerra, J. (2014). Filosofía Lean Construction para la gestión de proyectos de construcción: una revision actual. Avances de Investigación en Ingeniería, 11(1) 
Díaz-Bravo, L., Torruco-García, U., Martínez-Hernández, M., y Valera-Ruiz, M. (2013). La entrevista, recuerso flexible y dinámico. Investigación en educación médica, 2(7), 162-167

Espinal, A., Montoya, R., y Arenas, J. (2010). Gestión de almacenes y tecnologías de la información y comunicación (TIC). Estudios gerenciales, 26(117), 145-171.

Fernández, F. (2002). El análisis de contenido como ayuda metodológica para la investigación. Revista de Ciencias Sociales, 2(96), 35-53

López, M., Grajales, M., y Corrales, M. (2017). Lean construction-LC bajo pensamiento Lean. Revista Ingenierías de la Universidad de Medellín, 16(30), 115-128

Medina, V., y José, M. (2012). El servicio post venta y su incidencia en la fidelidad de los clientes de la Empresa Importadora Alvarado Vásconez Cía. Ltda., de la ciuidad de Ambato. Ambato
Olivos, P., Carrasco, F., Flores, J., Moreno, Y., y Nava, G. (2015). Modelo de gestión logística para pequeñas y medianas empresas en México. Contaduría y administrimación, 60(1), 181-203

Pedrosa, I., Juarros-Basterretxea, J., RoblesFernández, A., Basterio, J., y GarcíaCueto, E. (2015). Prueba de bondad de ajuste en distribuciones simétricas ¿qué estad'sitico utilizar? Universitas Psychologica, 14(1), 245-254

Ré, M., Arena, L., y Giubergia, M. (2012). Incorporación de TICs a la enseñanza de a Física. Laboratorios visturales basados en simulación. Revista Iberoamericana de Tecnología en Educación y Educación en Tecnologías, 8, 16-22

Trujillo, R., Llinás, H., Obeso, V., y Rojas, C. (2005). Influencia de la tecnología en el aprendizaje en el c'laculo diferencia y estadís descriptiva. Zona Próxima, 6, 94-105 